problems, and chest pain. In 9,686 (62.1\%) calls, patients were transported to a hospital.

Conclusion: This study showed that frequent users constitute a significant proportion of all EMS calls; they may explain the substantial increase seen in EMS call volumes in the last few years. Individually tailored interventions to users with an annual call volume of $\geq 10$ should be considered.

Prehosp Disaster Med 2017;32(Suppl. 1):s171-s172

doi:10.1017/S1049023X17004599

Prehospital Care at a Mass Endurance Event: The Chicago Marathon Experience

Anita V. Goyal ${ }^{1}$, Valentino Constantinou ${ }^{2}$, Jennifer Fokas ${ }^{3}$,

Sarah Van Duesen Phillips ${ }^{3}$, Jennifer Chan ${ }^{4}$,

George T. Chiampas ${ }^{4}$

1. Emergency Medicine, University of Illinois at Chicago, Chicago/ IL/United States of America

2. Industrial Engineering And Management Sciences, Northwestern University, Evanston/IL/United States of America

3. Chicago Event Management, Chicago/IL/United States of America

4. Emergency Medicine, Northwestern University, Chicago/IL/ United States of America

Study/Objective: To investigate the impact of prehospital care on patient outcomes and public health systems for the Chicago Marathon from 2012 to 2015.

Background: The Bank of America Chicago Marathon is a 26.2 mile race with approximately 40,000 runners annually. One of six World Marathon Majors, it serves as a model for mass event field medicine. The goals of this medical infrastructure are to decrease time to medical attention and transport, triage effectively, decrease hospital burden, and optimize public safety. This study examines impacts of medical structures at the Chicago Marathon, highlighting patient care volumes and trends.

Methods: A comprehensive retrospective study was conducted of the Chicago Marathon during 2012-2015. Prehospital medical care involved 21 medical aid stations on course, and two major medical tents in the finish area, including general care and intensive care units. Each facility, staffed with medical personnel, conducted its own triaging. Additionally, each site was equipped with an EMS Superior ambulance for transferring patients to a higher level of care if needed. Medical data was collected via paper and a digital Medical Patient Tracking System designed by Nika Tec.

Results: During 2012 to 2015, in total 4,963 people encountered pre-hospital care at the marathon. Of these, 175 people (3.53\%) were transferred to a hospital for further care (Figure 1). One-hundred-thirty (2.62\%) people were treated in the on-site ICU facilities, suggesting that the triage system imperatively connects patients to appropriate care without overburdening particular personnel and resources.

Conclusion: The majority of individuals seeking care were successfully triaged and treated by the personnel and resources on site, addressing the needs of an average of over 1,200 individuals per event. The small fraction transferred to hospitals minimized the public health burden, while identifying and mobilizing those who required transfer. This event can be

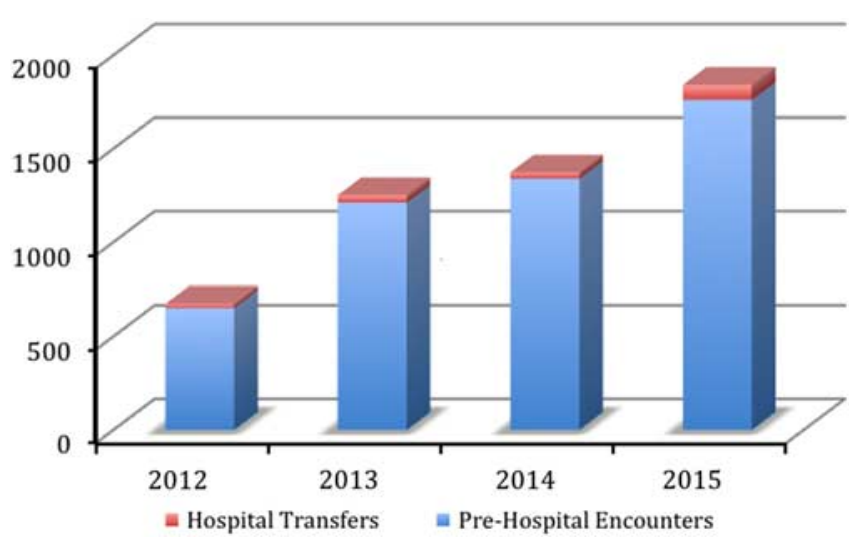

Figure 1. Number of Medical Encounters at Chicago Marathon 2012-2015.

likened to mass events both planned and unexpected, and it demonstrates strategic pre-hospital medical preparedness.

Prehosp Disaster Med 2017;32(Suppl. 1):s172

doi:10.1017/S1049023X17004605

\section{Analysis of Prehospital Scene Times and Interventions on Mortality Outcomes in Victims of Blunt and \\ Penetrating Trauma \\ Matthew D. Sztajnkrycer ${ }^{1}$, Octavia Ruelas ${ }^{2}$, Christine Lobse ${ }^{2}$ \\ 1. Emergency Medicine, Mayo Clinic, Rochester/MN/United States of America \\ 2. Mayo Clinic, Rochester/MN/United States of America}

Study/Objective: This study was to perform an outcome analysis of patients presenting after blunt (BT) and penetrating (PT) traumatic events.

Background: Recent studies have suggested improved outcomes in victims of penetrating trauma, managed with shorter prehospital times and limited interventions.

Methods: Descriptive analysis of the 2014 National Emergency Medical Services Information System (NEMSIS) public release research data set for patients presenting after acute traumatic injury.

Results: A total of 2,018,141 patient encounters met criteria, of which $3.9 \%$ were PT. Prehospital cardiac arrest occurred in 0.5\% BT patients, and $4.2 \%$ PT patients. $0.8 \% \mathrm{BT}$ patients and 4.1\% PT patients died in the ED. Scene times were 18.1 (IQR 11.0-21.0) minutes for BT and 16.0 (IQR 8.0-17.0) minutes for PT, while transport times were 15.1 (IQR 7.0-19.0) minutes and 14.4 (IQR 6.0-17.0) minutes for BT and PT, respectively. Mean scene time for BT patients who died in the ED was 24.9 (IQR 10.0-24.0) minutes compared with 18.8 (IQR 11.0-22.0) minutes for those admitted; for PT, scene times were 17.9 (IQR 8.0-18.0) and 13.4 (IQR 6.0-17.0) minutes, respectively. Mean number of procedures performed for BT patients who died in the ED was 6.5 (IQR 3.0-9.0) compared with 3.1 (IQR 1.0-4.0) for those who survived until admission; for PT, number of procedures performed were 5.7 (IQR 3.0-7.0) and 2.6 (IQR 1.0-3.0), respectively. 2.2\% $\mathrm{BT}$ and $14.8 \% \mathrm{PT}$ patients receiving prehospital venous access died in the ED. 